\title{
Model-Based Diagnosis and Prognosis of a Water Recycling System
}

\author{
Indranil Roychoudhury \\ SGT Inc. \\ NASA Ames Research Center \\ Moffett Field, CA 94035, USA \\ indranil.roychoudhury@nasa.gov
}

\author{
Vasyl Hafiychuk \\ SGT Inc. \\ NASA Ames Research Center \\ Moffett Field, CA 94035, USA \\ vasyl.hafiychuk@nasa.gov
}

\author{
Kai Goebel \\ NASA Ames Research Center \\ Moffett Field, CA 94035, USA \\ kai.goebel@nasa.gov
}

\begin{abstract}
A water recycling system (WRS) deployed at NASA Ames Research Center's Sustainability Base (an energy efficient office building that integrates some novel technologies developed for space applications) will serve as a testbed for long duration testing of next generation spacecraft water recycling systems for future human spaceflight missions. This system cleans graywater (waste water collected from sinks and showers) and recycles it into clean water. Like all engineered systems, the WRS is prone to standard degradation due to regular use, as well as other faults. Diagnostic and prognostic applications will be deployed on the WRS to ensure its safe, efficient, and correct operation. The diagnostic and prognostic results can be used to enable condition-based maintenance to avoid unplanned outages, and perhaps extend the useful life of the WRS. Diagnosis involves detecting when a fault occurs, isolating the root cause of the fault, and identifying the extent of damage. Prognosis involves predicting when the system will reach its end of life irrespective of whether an abnormal condition is present or not. In this paper, first, we develop a physics model of both nominal and faulty system behavior of the WRS. Then, we apply an integrated model-based diagnosis and prognosis framework to the simulation model of the WRS for several different fault scenarios to detect, isolate, and identify faults, and predict the end of life in each fault scenario, and present the experimental results.
\end{abstract}

\section{Table of Contents}

1 INTRODUCTION .......................... 1

2 Modeling the WATER RecyCling SyStem . 2

3 Diagnosis and Prognosis ApProach ....... 4

4 EXPERIMENTAL RESULTS .................. 7

5 Conclusions $\ldots \ldots \ldots \ldots \ldots \ldots \ldots \ldots \ldots \ldots \ldots \ldots, 8$

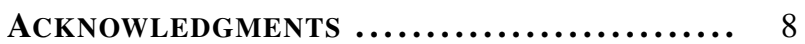

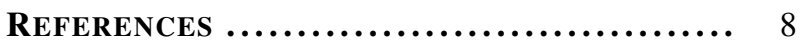

BIOGRAPHY .............................. 9

\section{INTRODUCTION}

The ability to recycle potable water from waste water is an integral part of the Environmental Control and Life Support System (ECLSS) of human-rated space missions. Several water recycling systems (WRSs) have been tested and deployed by NASA in the past, such as the Advanced Water Recovery System (AWRS) designed and built at the NASA Johnson Space Center (JSC) as part of the Advanced Life Support System [1], and the Direct Osmotic Concentration (DOC) System [2], currently undergoing performance testing at JSC.

978-1-4673-1813-6/13/\$31.00 (C)2013 IEEE.

1 IEEEAC Paper \#2344, Version 2, Updated 03/01/2013.
The WRS [3] deployed at NASA Ames Research Center's Sustainability Base [4] - a Leadership in Energy and Environmental Design (LEED) certified energy efficient office building built to, among other things, put cutting-age space technologies to work on Earth - has been developed to serve as a testbed for long duration testing of next generation spacecraft water recycling systems. This system cleans graywater (human waste water collected from sinks and showers) and recycles it into clean water to be used as flush water in the Sustainability Base with the goal of reducing the water comsumption of the building by $60 \%$. The WRS is mainly comprised of a forward osmosis (FO) system and a reverse osmosis ( $\mathrm{RO})$ system. In the FO system, the graywater is separated from saltwater through semi-permeable membranes, and water moves through the semi-permeable membranes from a region of higher water chemical potential (i.e., graywater) to a region of lower water chemical potential (i.e., saltwater). In the RO system, hydraulic pressure is applied to the (now dilute) saltwater to force water from a region of lower water chemical potential (i.e., saltwater) to a region of higher water chemical potential (i.e., clean product water) through another set of semi-permeable membranes, thereby extracting clean water.

The WRS is a complex hydraulic system with a large number of components. Complex engineered systems are subject to degradation even in regular use (as well as the possibility of incurring faults) and the WRS is no exception. Hence, diagnosis and prognosis applications will increasingly be implemented on future engineered systems to ensure their safe, efficient, and correct operation. The diagnostic and prognostic results can be used to enable condition-based maintenance to avoid unplanned outages, and perhaps extend the useful life of the system. Diagnosis involves detecting when a fault occurs, isolating the root cause of the fault, and identifying the extent of damage. Prognosis involves prediction of when the system will reach its end of (useful) life so that mitigating actions may be implemented.

In this paper, we apply a model-based diagnosis and prognosis framework [5] on the WRS. We generate a physics model of the nominal and faulty system behavior that captures the dynamics of the WRS in the hydraulic domain, as well as the concentration of solute in the system. Faults are modeled as unexpected changes in the system parameters. We assume the presence of only single, persistent faults but allow faults of different fault magnitudes. As the system operates, the observed measurements are compared to estimates of nominal measurements obtained from the nominal system model, and a statistically significant measurement deviation from nominal results in a fault to be detected. Then, as measurements deviate, the observed measurement deviations are compared to predictions of how each measurement should deviate given particular faults, and any fault that is inconsistent with the observed measurement deviations is 


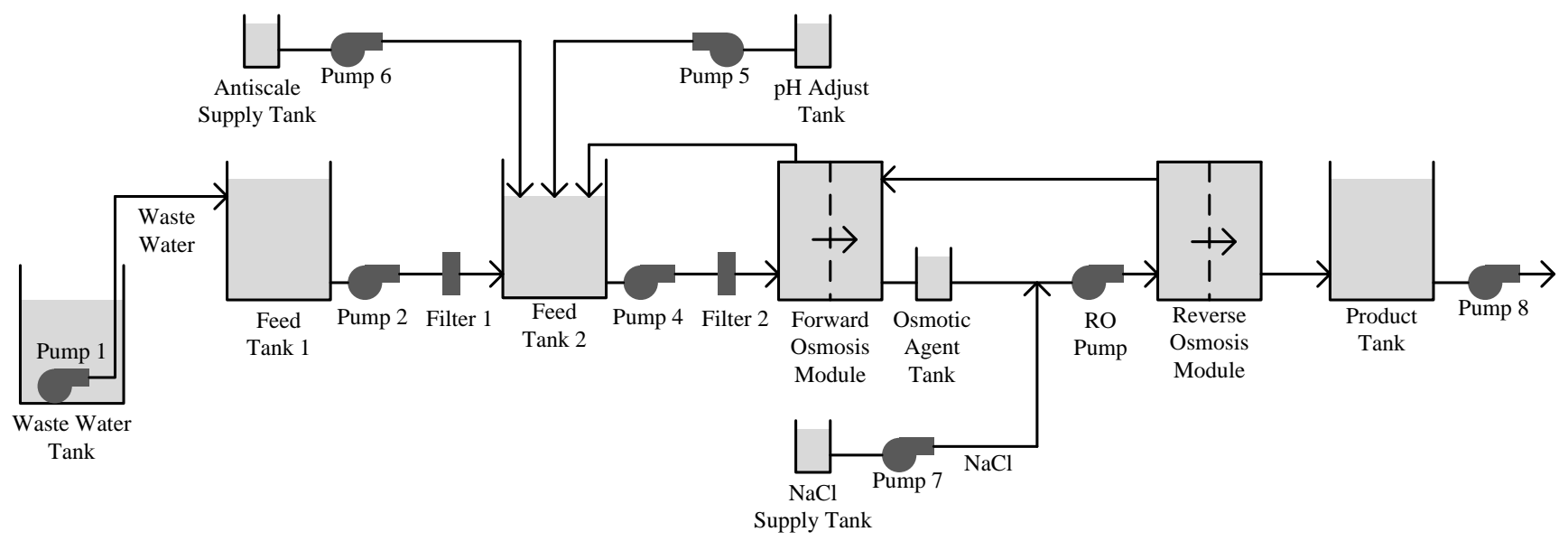

Figure 1. Schematic of the complete Water Recycling System.

removed from consideration. For fault identification, once the number of fault candidates is reduced to less than a predefined number, for each fault candidate, a hypothesized fault model for that particular fault candidate is generated, and joint state-parameter estimation is performed [6]. For prognosis, the end of life of the system is predicted, using, for each hypothesized fault candidate, a predictor based on a fault progression model integrated with the nominal model [7]. Finally, we present results of several diagnosis and prognosis experiments performed on the simulation model of the WRS.

The paper is organized as follows. Section 2 presents the nominal and faulty system model of the WRS. Section 3 describes the diagnosis and prognosis approach used in this work. Experimental results are presented in Section 4, and Section 5 concludes the paper.

\section{Modeling the WATER RECyCling SYSTEM}

The WRS installed at the Sustainablity Base at NASA Ames Research Center uses osmosis for generating clean water from waste water. As mentioned earlier, the WRS is mainly comprised of a forward osmosis (FO) module and a reverse osmosis (RO) module. FO is the movement of solvent molecules (in our case, water) across a semi-permeable membrane from a region of higher water chemical potential (usually called the feed solution) to a region of lower water chemical potential (usually called the osmotic agent) [8]. RO, on the other hand, is the movement of solvent molecules across a semi-permeable membrane in the opposite direction of FO, i.e., from a region of lower water chemical potential to a region of higher water chemical potential due to the application of hydraulic pressure.

Osmosis is driven by the difference in solute concentrations across the membrane that allows the solvent molecules to pass, but rejects most solute molecules and ions. The general equation describing water transport in $\mathrm{FO}$ and $\mathrm{RO}$ is

$$
J_{w}=A(\sigma \Delta \pi-\Delta P)
$$

where, $J_{w}$ is the water flux (rate of flow of water per unit cross sectional area), $A$ is the water permeability constant of the membrane (i.e., the measure of the transport flux of material through the membrane per unit driving force per unit membrane thickness), $\sigma$ is the reflection coefficient (i.e., measure of how much a membrane can "reflect" solute particles from passing through), $\Delta \pi$ is the osmotic pressure differential, and $\Delta P$ is the applied (hydraulic) pressure differential. Osmotic pressure is the pressure that would prevent the transport of solvent across the membrane, when applied to the more concentrated solution. The driving force in FO is the osmotic pressure differential across the membrane $(\Delta \pi)$, while in $\mathrm{RO}$, the applied hydraulic pressure differential $(\Delta P)$ that opposes and exceeds the osmotic pressure differential to force water from a region of lower water chemical potential to a region of higher water chemical potential across the membrane. The hydraulic pressure is generated by pumps that are responsible for maintaining the needed pressure differential. Therefore, in Eqn. $1, \Delta P \approx 0$ for $\mathrm{FO}$ and $\Delta P>\Delta \pi$ for $\mathrm{RO}$.

Fig. 1 presents a schematic of the WRS, which consists of several tanks, pumps, pipes, filters, and the FO and RO modules. During nominal operation, first, Pump 1 is switched on to pump water from the Waste Water Tank into Feed Tank 1 till the latter is full. Then, Pump 1 shuts off and Pump 2 is turned on to fill Feed Tank 2. Filter 1 between Pump 2 and Feed Tank 2 traps suspended solids in the feed solution and prevents them from entering Feed Tank 2. Pump 2 runs till Feed Tank 2 is full. Pumps 5 and 6 are small diaphragm metering pumps that are turned on periodically to add antiscale chemicals (from the Antiscale Supply Tank) to the feed and adjust its $\mathrm{pH}$ (by adding chemicals from the $\mathrm{pH}$ Adjust Tank), respectively. Then Pump 4 is powered on to recirculate the feed water through Filter 2, and the FO module back to the Feed Tank 2. The osmotic agent is stored in the Osmotic Agent (OA) Tank. The OA in the WRS is a salt $(\mathrm{NaCl})$ solution. The concentration of OA determines the rate of flow of water. The goal is to maintain this flow at approximately $155 \mathrm{Lh}^{-1}$. However, during the nominal operation of the WRS, some $\mathrm{NaCl}$ is lost through the membranes. Hence, additional $\mathrm{NaCl}$ is added to the $\mathrm{OA}$ to maintain the flow of water through the membrane. The initial concentration of OA is $10 \mathrm{gL}^{-1}$, but the controller can add up to $20 \mathrm{gL}^{-1}$ of additional $\mathrm{NaCl}$ solution to the $\mathrm{OA}$ from the $\mathrm{NaCl}$ Supply Tank. The RO module applies an external pressure to maintain the flow of water through the RO membrane to approx $155 \mathrm{Lh}^{-1}$. The Reverse Osmosis (RO) pump recirculates the diluted OA between the RO and the FO modules. Clean water from the RO Module is collected in the Product Tank. The WRS is operated in a semi-batch mode, with no extra feed added to Feed Tank 2 once the FO and RO modules are started till $95 \%$ 


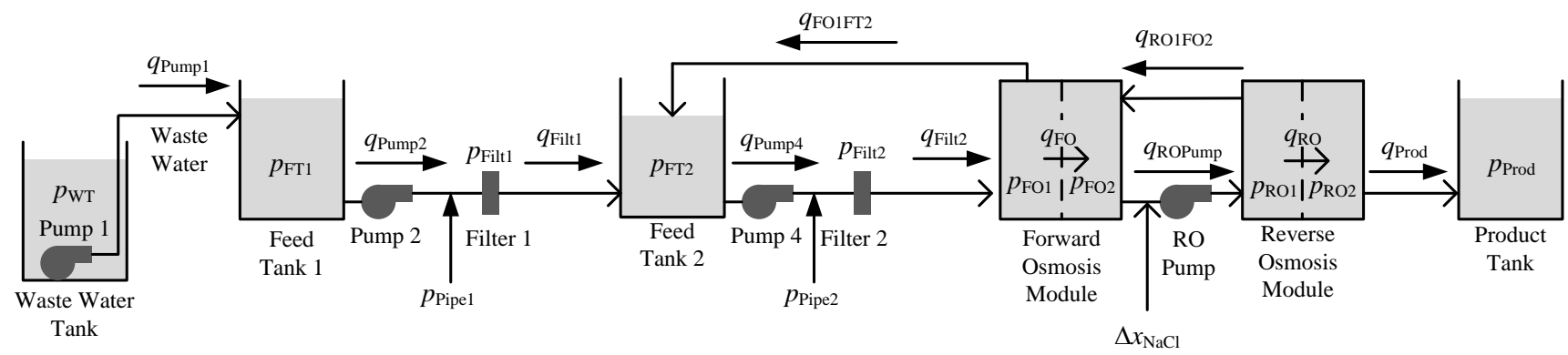

Figure 2. Schematic of the subset of Water Recycling System and sensors for experiments.

of clean water is recovered from the feed water through $\mathrm{FO}$ and $\mathrm{RO}$, after which, the remaining waste is disposed.

In this paper, we apply our diagnosis and prognosis scheme to a subset of the WRS, as shown in Fig. 2. This subset consists of all components of the complete WRS except the Antiscale Supply Tank, pH Adjust Tank, the $\mathrm{NaCl}$ Supply Tank, and Pumps $5-7$. These pumps are only on for short durations before the FO and RO modules are activated, and omitting these and the associated tanks does not adversely alter the main dynamics of the WRS. Note that in Fig. 2, the Osmotic Agent Tank is also not considered, and instead, the $\mathrm{OA}$, i.e., $\mathrm{NaCl}$, is assumed to be added directly in the FO-RO recirculation path. Moreover, Pump 8 is not turned on during the simulation, and it is also omitted in Fig. 2.

\section{Nominal Modeling}

We develop the nominal system model for the WRS using the state space formulation:

$$
\begin{aligned}
& \dot{\mathbf{x}}(t)=\mathbf{f}(t, \mathbf{x}(t), \boldsymbol{\theta}(t), \mathbf{u}(t), \mathbf{v}(t)) \\
& \mathbf{y}(t)=\mathbf{h}(t, \mathbf{x}(t), \boldsymbol{\theta}(t), \mathbf{u}(t), \mathbf{n}(t)),
\end{aligned}
$$

where $t \in \mathbb{R}$ denotes continuous time, $\mathbf{x}(t) \in \mathbb{R}^{n_{x}}$ is the state vector, $\boldsymbol{\theta}(t) \in \mathbb{R}^{n_{\theta}}$ is the parameter vector, $\mathbf{u}(t) \in \mathbb{R}^{n_{u}}$ is the input vector, $\mathbf{v}(t) \in \mathbb{R}^{n_{v}}$ is the process noise vector, $\mathbf{f}$ is the state equation, $\mathbf{y}(t) \in \mathbb{R}^{n_{y}}$ is the output vector, $\mathbf{n}(t) \in \mathbb{R}^{n_{n}}$ is the measurement noise vector, and $\mathbf{h}$ is the output equation. The parameters $\boldsymbol{\theta}(t)$ are typically considered as constants in the nominal system model.

Our physics-based lumped-parameter model of the WRS represents its hydraulic dynamics. In the hydraulic domain, we denote volumetric flow rate as $q$ and hydraulic pressure as $p$. The pressures are the state variables in our model. As shown in Fig. 2, the pressures at the bottom of the Waste Water Tank, Feed Tank 1, Feed Tank 2, and Product Tank are denoted by $p_{\mathrm{WT}}, p_{\mathrm{FT} 1}, p_{\mathrm{FT} 2}$, and $p_{\text {Prod }}$, respectively. The FO (resp. RO) module is modeled as two tanks, FO1 and FO2 (resp. RO1 and RO2) ${ }^{2}$, with pressures $p_{\mathrm{FO} 1}$ and $p_{\mathrm{FO} 2}$ (resp. $p_{\mathrm{RO} 1}$ and $p_{\mathrm{RO} 2}$ ), are separated by the FO (resp. RO) membrane, and $p_{\mathrm{FO}}$ (resp. $p_{\mathrm{RO}}$ ) denotes the volumetric flow rate of water across the FO (resp. RO) membrane. The pipe segments Pipe 1 and Pipe 2, between Pump 2 and Filter 1, and Pump 4 and Filter 2, respectively, are also modeled as very small tanks, with pressures, $p_{\text {Pipe1 }}$ and $p_{\text {Pipe } 2}$, respectively.

The outflow rate of Pump 1, Pump 2, Pump 4, and RO Pump are denoted by $q_{\text {Pump1 }}, q_{\text {Pump2 }}, q_{\text {Pump } 4}$, and $q_{\text {ROPump }}$, respectively. The flow through Filter 1 and Filter 2 are

${ }^{2}$ Labels FO1, FO2, RO1 and RO2 are omitted from Fig. 2 for clarity. denoted by $q_{\text {Filt1 }}$ and $q_{\text {Filt2 }}$, respectively, and $q_{\text {Prod }}$ denotes the rate of flow of clean water into the Product Tank from the RO Module. $q_{\mathrm{FO} 1 \mathrm{FT} 2}$ denotes the flow of water from the FO module to Feed Tank 2 , and $q_{\mathrm{RO} 1 \mathrm{FO} 2}$ denotes the flow of water from the RO module to the FO module.

A Pump $j$ installed between two points with pressures $q_{i}$ and $q_{j}$, respectively, is modeled to boost the pressure at its input by its boost pressure $p_{\text {Pumpj }}$, i.e., the pressure difference between these two points with the pump in between is $p_{i}+$ $p_{\text {Pumpj }}-p_{j}$. The boost pressure $p_{\text {Pumpj }}$ is considered as an input to the system. The boost pressures for Pump 1, Pump 2, Pump 4 , and RO Pump are denoted by $p_{\text {Pump } 1}, p_{\text {Pump2 }}, p_{\text {Pump } 4}$, and $p_{\text {ROPump }}$, respectively.

Given two points in a hydraulic system, with pressures $p_{i}$ and $p_{j}$, the volumetric flow rate of fluid between these two points is

$$
q_{i j}=R_{i j} \sqrt{\left|p_{i}-p_{j}\right|} \operatorname{sign}\left(p_{i}-p_{j}\right),
$$

where $R_{i j}$ is the coefficient of flow for $q_{i j}$. For a Tank $i$ having input and output flow rates, $q_{\text {in }}$ and $q_{\text {out }}$, pressure $p_{\text {Tanki }}$ is

$$
\dot{p}_{\text {Tanki }}=\frac{1}{C_{\text {Tanki }}}\left(q_{\text {in }}-q_{\text {out }}\right),
$$

where $C_{\text {Tanki }}$ is the tank capacitance.

In addition to the hydraulic dynamics, we also model the reduction of solute molecules in the OA over time. To this end, the amount of $\mathrm{NaCl}$ in the $\mathrm{OA}, x_{\mathrm{NaCl}}$, is considered a state variable. As mentioned before, we start with $10 \mathrm{gL}^{-1}$ of $\mathrm{NaCl}$ in the OA. During nominal operation of the WRS, some $\mathrm{NaCl}$ is lost through the membranes (we assume the rate of loss of salt to be $-1.11 \times 10^{-5} \mathrm{gL}^{-1} \mathrm{~s}^{-1}$ ). Now, the osmotic potential $\Delta \pi$ is directly proportional to the difference in concentration on the two sides of the semi-permeable membrane. Also, the goal of the controller is to maintain the flow through the FO membrane at approximately $155 \mathrm{Lh}^{-1}$. To maintain the osmotic pressure difference, and hence, the rate of flow of water through the FO membrane, the controller adds additional amounts of $\mathrm{NaCl}$, represented by $\Delta_{x_{\mathrm{NaCl}}}$, to the OA. However, the total amount of $\mathrm{NaCl}$ in the $\mathrm{OA}$ cannot be more than $30 \mathrm{gL}^{-1}$, and hence the maximum value of $\Delta_{x_{\mathrm{NaCl}}}$ can be $20 \mathrm{gL}^{-1}$. This $\Delta_{x_{\mathrm{NaCl}}}$ also affects the flow of water through the RO membrane.

Fig. 3 lists the equations that model the WRS, where the state variables include $\mathbf{x}=\left[p_{\mathrm{WT}}, p_{\mathrm{FT} 1}, p_{\text {Pipe } 1}, p_{\mathrm{FT} 2}, p_{\mathrm{Pipe} 2}, p_{\mathrm{FO} 1}\right.$, $\left.p_{\mathrm{FO} 2}, p_{\mathrm{RO} 1}, p_{\mathrm{RO} 2}, p_{\mathrm{Prod}}, x_{\mathrm{NaCl}}\right]^{T}$; the output variables include 
$\mathbf{y}=\left[q_{\text {Pump2 } 2}, q_{\text {Filt1 }}, q_{\text {Filt2 }}, q_{\text {Pump4 }}, q_{\text {ROPump }}, q_{\text {Prod }}, p_{\mathrm{WT}}, p_{\mathrm{FT} 1}\right.$, $\left.p_{\text {FT2 }}, p_{\text {Prod }}, p_{\text {Filt1 }}, p_{\text {Filt2 }}\right]^{T}$; and the input variables include $\mathbf{u}=\left[u_{\text {Pump1 }}, u_{\text {Pump2 }}, u_{\text {Pump } 4}, u_{\text {ROPump }}, u_{\mathrm{FO}}, u_{\mathrm{RO}}\right]^{T}$. The input signals switch the corresponding pump on or off. The input signals $u_{\mathrm{FO}}$ and $u_{\mathrm{RO}}$ basically indicate when the FO and RO modules are filled with feed and osmotic agent (we assume there is no water in any of the tanks or plumbings at the start of the simulation), and hence, FO and RO can begin, respectively. All flows are expressed in $\mathrm{Lh}^{-1}$ and all pressures are expressed in psi.

\section{Modeling of Faulted System}

Typical degradation modes of the WRS include clogged membranes, clogged filters, and sensor faults. In particular, Filter 1, Filter 2, the FO membrane, and the RO membrane all get clogged over time due to buildup of solids. These clogging faults, denoted by $R_{\mathrm{Filt} 1}^{-}, R_{\mathrm{Filt} 2}^{-}, A_{\mathrm{FO}}^{-}$, and $A_{\mathrm{RO}}^{-}$, respectively, are represented as gradual decrease in the coefficient of flow through the filters, $R_{\text {Filt } 1}$ and $R_{\text {Filt } 2 \text {, and the }}$ membrane permeabilities $A_{\mathrm{FO}}$ and $A_{\mathrm{RO}}$, respectively. A fault can then be modeled as an unexpected change in a system parameter. For Filter $i$, the gradual decrease in $R_{\text {Filti }}$ is represented as

$$
\dot{R}_{\text {Filti }}= \begin{cases}0, & t<t_{f} \\ \Delta R_{\text {Filti }}, & \text { otherwise }\end{cases}
$$

where $t_{f}$ is the time of fault occurrence, and $\Delta R_{\text {Filt1 }}$ is the fault parameter. Similarly, for membrane $j$, the gradual decrease in $A_{i}$ is represented as

$$
\dot{A}_{i}= \begin{cases}0, & t<t_{f} \\ \Delta A_{i}, & \text { otherwise }\end{cases}
$$

where $\Delta A_{i}$ is the fault parameter.

Sensors faults can include abrupt bias and gradual drift fault. A bias fault in sensor $S$ is indicated as $S^{(b, \Delta S)}$, and is modeled as an abrupt addition of a constant bias $b$ added to the sensor value from the point of fault injection $t_{f}$, i.e.,

$$
S= \begin{cases}S, & t<t_{f} \\ S+\Delta S, & \text { otherwise. }\end{cases}
$$

A drift fault in sensor $S$ is indicated as $S^{(d, \Delta S)}$, and is modeled as a gradual addition of a constant drift $d$ to the sensor value at each time step from the point of fault injection $t_{f}$, i.e.,

$$
\dot{S}= \begin{cases}0, & t<t_{f} \\ \Delta S, & \text { otherwise }\end{cases}
$$

The sensor faults considered in this paper include $q_{\text {Filt1 }}^{(b, 20)}$, $q_{\text {Filt1 }}^{(d, 0.1)}, p_{\text {Prod }}^{(b, 2)}$, and $q_{\text {ROPump }}^{(d, 0.01)}$.

\section{Diagnosis and Prognosis ApProach}

Fig. 4 illustrates the architecture of our diagnostic and prognostic approach, which is adopted from that presented in [5]. At each discrete time step, $k$, the system takes as inputs $\mathbf{u}(k)$, and outputs measurements $\mathbf{y}(k)$. The nominal model observer also takes as inputs $\mathbf{u}(k)$, and generates estimates of nominal measurements, $\hat{\mathbf{y}}(k)$. The fault detector then takes in the observed and estimated measurements, $\mathbf{y}(k)$ and $\hat{\mathbf{y}}(k)$, and detects when a fault has occurred based on the residual, $\mathbf{r}(k)=\mathbf{y}(k)-\hat{\mathbf{y}}(k)$. Once a fault is detected, fault isolation is initiated. The fault isolation block takes as inputs $\mathbf{r}(k)$. These measurement residuals are used along with predictions of how each measurement is expected to deviate from nominal for each possible fault in the system to generate a set of fault candidates $F(k)$ at time $k$ that explain the observed deviations in measurements till time $k$. The fault identification module, for each fault, $f \in F(k)$, estimates $p\left(\mathbf{x}_{f}(k), \boldsymbol{\theta}_{f}(k) \mid \mathbf{y}(0: k)\right)$, where $\mathbf{x}_{f}$ represents the set of state variables in the faulty system model that includes all state variables of the nominal model and the faulty system parameter corresponding to the particular $f \in F(k)$ that needs to be estimated. $\boldsymbol{\theta}_{f}$ represents the set of all original system parameter except those that are now included in $\mathbf{x}_{f}$ and includes some additional fault progression model parameters that are used to model how the faulty parameter progresses over time (see [5] for details). Finally, the prediction module takes as input $p\left(\mathbf{x}_{f}(k), \boldsymbol{\theta}_{f}(k) \mid \mathbf{y}(0: k)\right)$ to make predictions of End of Life (EOL), i.e., $p\left(\mathrm{EOL}_{f}(k) \mid y(0: k)\right)$, and Remaining Useful Life (RUL), i.e., $p\left(\operatorname{RUL}_{f}(k) \mid y(0: k)\right)$ [7].

A system is said to have reached its EOL when one or more constraints that define the acceptable behavior of the system is violated. For each faulty system model, we define a threshold function, $T_{\mathrm{EOL}_{f}}$, where $T_{\mathrm{EOL}_{f}}\left(\mathbf{x}_{f}(t), \boldsymbol{\theta}_{f}(t)\right)=1$ if these constraints are violated, and $T_{\mathrm{EOL}_{f}}\left(\mathbf{x}_{f}(t), \boldsymbol{\theta}_{f}(t)\right)=0$ otherwise. So, $\mathrm{EOL}_{f}$ may be defined as $\mathrm{EOL}_{f}\left(t_{P}\right) \triangleq$ $\inf \left\{t \in \mathbb{R}: t \geq t_{P}\right.$ and $\left.T_{\mathrm{EOL}_{f}}\left(\mathbf{x}_{f}(t), \boldsymbol{\theta}_{f}(t)\right)=1\right\}$, i.e., EOL is the earliest time point at which the threshold is reached. Given $\operatorname{EOL}_{f}\left(t_{P}\right)$, RUL may then be defined with $\mathrm{RUL}_{f}\left(t_{P}\right) \triangleq \mathrm{EOL}_{f}\left(t_{P}\right)-t_{P}$. The remainder of this section describes the details of the different modules of the integrated diagnosis and prognosis architecture.

\section{Nominal Model Observer}

The nominal model observer typically takes as inputs the system inputs, $\mathbf{u}(k)$, and measurements, $\mathbf{y}(0: k)$, and the initial state of the system, and uses the state transition function, $\mathbf{f}(\cdot)$, and observation function, $\mathbf{h}(\cdot)$, to estimate distributions of states, $\mathbf{x}(k)$, and parameters, $\boldsymbol{\theta}(k)$, i.e., $p(\mathbf{x}(k), \boldsymbol{\theta}(k) \mid \mathbf{y}(0: k))$. Any appropriate filtering scheme, e.g., Kalman filter, extended Kalman filter, unscented Kalman filter, particle filter [9], among others, can be adopted as the nominal observer. Note that in this paper, a high fidelity simulation model of the nominal WRS system developed using the equations shown in Fig. 3 is used in place of the nominal observer to simulate the nominal system behavior given the inputs $\mathbf{u}$ and initial state of the system.

\section{Fault Detection}

A fault is detected when a residual, $r(k) \in \mathbf{r}(k)$, i.e., the difference between the observed (faulty) and estimated (nominal) values of a measurement, is determined to be statistically significant [10]. In our work, we use a $Z$-test coupled with a sliding window technique to determine this statistical significance [10]. Fault detectors need to be tuned so as to minimize false alarms and missed detections while maintaining the desired level of sensitivity.

\section{Fault Isolation}

Once a fault is detected, at each subsequent time step, every measurement residual is qualitatively abstracted into a tuple of qualitative symbols, $\left(\sigma_{1}, \sigma_{2}\right)$, where $\sigma_{1} \in\{0,+,-\}$ rep- 


$$
\begin{aligned}
& p_{\mathrm{WT}}=\frac{1}{C_{\mathrm{WT}}}\left(-q_{\mathrm{Pump} 1}\right) \\
& p_{\mathrm{FT} 1}=\frac{1}{C_{\mathrm{FT} 1}}\left(q_{\mathrm{Pump} 1}-q_{\mathrm{Pump} 2}\right) \\
& p_{\text {Pipe1 }}=\frac{1}{C_{\text {Filt1 }}}\left(q_{\text {Pump2 }}-q_{\text {Filt1 }}\right) \\
& p_{\mathrm{FT} 2}=\frac{1}{C_{\mathrm{FT} 2}}\left(q_{\mathrm{Filt} 1}+q_{\mathrm{FO} 1 \mathrm{FT} 2}-q_{\mathrm{Pump} 4}\right) \\
& p_{\text {Pipe2 }}=\frac{1}{C_{\text {Filt2 }}}\left(q_{\text {Pump4 }}-q_{\text {Filt2 }}\right) \\
& p_{\mathrm{FO} 1}=\frac{1}{C_{\mathrm{FO} 1}}\left(q_{\mathrm{Fil} 2}-q_{\mathrm{FO} 1 \mathrm{FT} 2}-q_{\mathrm{FO}}\right) \\
& p_{\mathrm{FO} 2}=\frac{1}{C_{\mathrm{FO} 2}}\left(q_{\mathrm{FO}}+q_{\mathrm{RO} 1 \mathrm{FO} 2}-q_{\mathrm{ROPump}}\right) \\
& p_{\mathrm{RO} 1}=\frac{1}{C_{\mathrm{RO} 1}}\left(q_{\mathrm{ROPump}}-q_{\mathrm{RO} 1 \mathrm{FO} 2}-q_{\mathrm{RO}}\right) \\
& p_{\mathrm{RO} 2}=\frac{1}{C_{\mathrm{RO} 2}}\left(q_{\mathrm{RO}}-q_{\text {Prod }}\right) \\
& p_{\text {Prod }}=\frac{1}{C_{\text {Prod }}}\left(q_{\text {Prod }}\right) \\
& x_{\mathrm{NaCl}}=-1.1111 \times 10^{-5} \\
& \Delta_{x_{\mathrm{NaCl}}}=\min \left(20, \frac{155 \times 2.78 \times 10^{-8}}{0.841 \times 10^{5} \times A_{\mathrm{FO}}}-x_{\mathrm{NaCl}}\right) \\
& q_{\text {Pump1 }}=u_{\text {Pump1 }}\left(R_{\text {Pump1 }} \sqrt{\left|p_{\mathrm{WT}}+p_{\text {Pump1 }}-p_{\mathrm{FT} 1}\right|} \operatorname{sign}\left(p_{\mathrm{WT}}+p_{\mathrm{Pump} 1}-p_{\mathrm{FT} 1}\right)\right) \\
& q_{\text {Pump2 }}=u_{\text {Pump2 }}\left(R_{\text {Pump2 }} \sqrt{\left|p_{\text {FT1 }}+p_{\text {Pump2 }}-p_{\text {Filt1 }}\right|} \operatorname{sign}\left(p_{\text {FT1 }}+p_{\text {Pump2 }}-p_{\text {Filt1 } 1}\right)\right) \\
& q_{\text {Filt1 }}=R_{\text {Filt1 }} \sqrt{\left|p_{\text {Pipe } 1}-p_{\text {FT2 } 2}\right|} \operatorname{sign}\left(p_{\text {Pipe } 1}-p_{\text {FT2 }}\right) \\
& q_{\text {Pump4 }}=u_{\text {Pump } 4}\left(R_{\text {Pump } 4} \sqrt{\left|p_{\text {FT2 }}+p_{\text {Pump } 4}-p_{\text {Filt2 }}\right|} \operatorname{sign}\left(p_{\text {FT2 }}+p_{\text {Pump } 4}-p_{\text {Filt2 }}\right)\right) \\
& q_{\mathrm{FO} 1 \mathrm{FT} 2}=R_{\mathrm{FO} 1 \mathrm{FT} 2} \sqrt{\left|p_{\mathrm{FO} 1}\right|} \operatorname{sign}\left(p_{\mathrm{FO} 1}\right) \\
& q_{\mathrm{Filt} 2}=R_{\mathrm{Filt} 2} \sqrt{\left|p_{\mathrm{Pipe} 2}-p_{\mathrm{FO} 1}\right|} \operatorname{sign}\left(p_{\mathrm{Pipe} 2}-p_{\mathrm{FO} 1}\right) \\
& q_{\mathrm{FO}}=u_{\mathrm{FO}} \cdot \operatorname{Area}_{\mathrm{FO}} \cdot A_{\mathrm{FO}}\left(x_{\mathrm{NaCl}}+\Delta_{x_{\mathrm{NaCl}}}\right) \times 0.841 \times 10^{5} \\
& \left.q_{\mathrm{RO} 1 \mathrm{FO} 2}=R_{\mathrm{RO} 1 \mathrm{FO} 2} \sqrt{\left|p_{\mathrm{RO} 1}-p_{\mathrm{FO} 2}\right|} \operatorname{sign}\left(p_{\mathrm{RO} 1}-p_{\mathrm{FO} 2}\right)\right) \\
& q_{\mathrm{ROPump}}=u_{\mathrm{ROPump}}\left(R_{\mathrm{ROPump}} \sqrt{\left|p_{\mathrm{FO} 2}+p_{\mathrm{ROPump}}-p_{\mathrm{RO} 1}\right|} \operatorname{sign}\left(p_{\mathrm{FO} 2}+p_{\mathrm{ROPump}}-p_{\mathrm{RO} 1}\right)\right) \\
& q_{\mathrm{RO}}=u_{\mathrm{RO}} \cdot \text { Area }_{\mathrm{RO}} \cdot A_{\mathrm{RO}}\left(20 \times 0.841 \times 10^{5}-\left(x_{\mathrm{NaCl}}+\Delta_{x_{\mathrm{NaCl}}}\right) \times 0.841 \times 10^{5}\right) \\
& q_{\text {Prod }}=R_{\text {Prod }} \sqrt{\left|p_{\text {RO2 }}-p_{\text {Prod }}\right|} \operatorname{sign}\left(p_{\text {RO2 }}-p_{\text {Prod }}\right) \\
& p_{\text {Filt1 }}=p_{\text {Pipe } 1}-p_{\text {FT2 }} \\
& p_{\text {Filt2 }}=p_{\text {Pipe2 }}-p_{\text {FO1 }}
\end{aligned}
$$

Figure 3. Equations of the nominal WRS model.

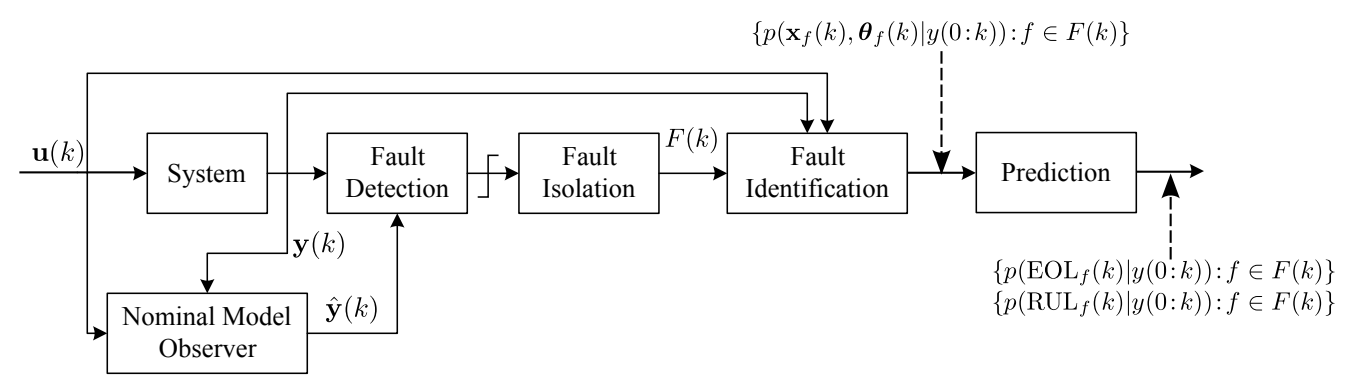

Figure 4. Diagnosis and Prognosis Architecture.

resents the qualitative magnitude change, and $\sigma_{2} \in\{0,+,-\}$ represents the qualitative slope change. The symbols, 0, + , or - , denote whether the magnitude or slope of this measurement is at, above, or below nominal, respectively. The symbols are generated using a sliding window technique as described in detail in [10]. 
Based on the first observed statistically significant measurement deviation, we generate a set of possible fault candidates. Then, for each fault candidate, we systematically determine a fault signature for each measurement [11]. A fault signature of a fault for a measurement is a prediction of how the measurement will deviate from nominal due to the fault. Fault signatures are also of the form $\left(s_{1}, s_{2}\right)$, where $s_{1} \in\{0,+,-\}$ and $s_{2} \in\{0,+,-\}$ capture qualitatively the direction of change to be expected in the magnitude and slope of each measurement from nominal if the fault occurs.

Given the set of fault candidates, as measurements deviate from nominal, the observed measurement deviations (captured symbolically) are checked for consistency with predicted fault signatures and measurement orderings. Any fault candidate whose predictions are inconsistent is removed from consideration. As more and more measurement deviations are observed, the candidate set will reduce, ideally resulting in a singleton.

However, in some cases, the qualitative fault signatures alone are not sufficient in distinguishing all faults, or fault effects may take too long to manifest, and quantitative analysis is needed to correctly diagnose the true fault. The advantage of using qualitative fault isolation is that it reduces the fault candidates very quickly, thereby improving the scalability of the overall diagnosis task. Hence, the more diagnosable the system is, the smaller is the number of possible fault candidates remaining after fault isolation is performed, and fewer will be the faults that will have to be isolated through relatively (computationally) expensive quantitative methods.

\section{Fault Identification}

We initiate quantitative fault identification after qualitative fault signature-based isolation is executed for $p$ time steps or till the number of fault candidates reduces to less than $\sigma$, whichever is achieved first. The design parameters $p$ and $\sigma$ are chosen based on the design requirements of the integrated diagnostic and prognostic system.

Once fault identification is invoked, under the single fault assumption, for each remaining fault candidate, $f$, we instantiate an observer using its faulty system model by extending the nominal system model with the fault progression model. Then each fault observer tracks the observed system measurements independently, and generates estimates of $\hat{\mathbf{y}}(k)$ and $p\left(\mathbf{x}_{f}(k), \boldsymbol{\theta}_{f}(k) \mid \mathbf{y}\left(k_{d}-\Delta k^{\max }: k\right)\right), \Delta k^{\max }$ is usually assumed to be larger than the time difference between the time of fault occurrence, $k_{f}$, and the time of fault detection, $k_{d}$. Each fault observer is initialized to estimated values of $\mathbf{x}$ and $\boldsymbol{\theta}$ obtained from the nominal observer at time $k_{d}-\Delta k^{\max }$, and the fault parameters are initialized to zero. If multiple fault candidates remain when fault identification is invoked, for each fault observer, a $Z$-test is used to determine if the deviation of a measurement estimated by the observer from the corresponding actual observation is statistically significant. Since we are considering only single faults, the expectation is that eventually, the estimates of only the correct fault observer will converge to the observed measurements, while those of all others will deviate from the observed measurements. Thus fault identification also helps in fault isolation. Practically, even the true fault model will take some time before tracking the measurements correctly, since initially, the fault parameter values are most likely to be not tuned to their true values. We assume that the true fault observer will converge to the observed measurements within $s_{d}$ time steps of its invocation. Thus, the $Z$-tests are monitored only after $s_{d}$ time steps are over [6].

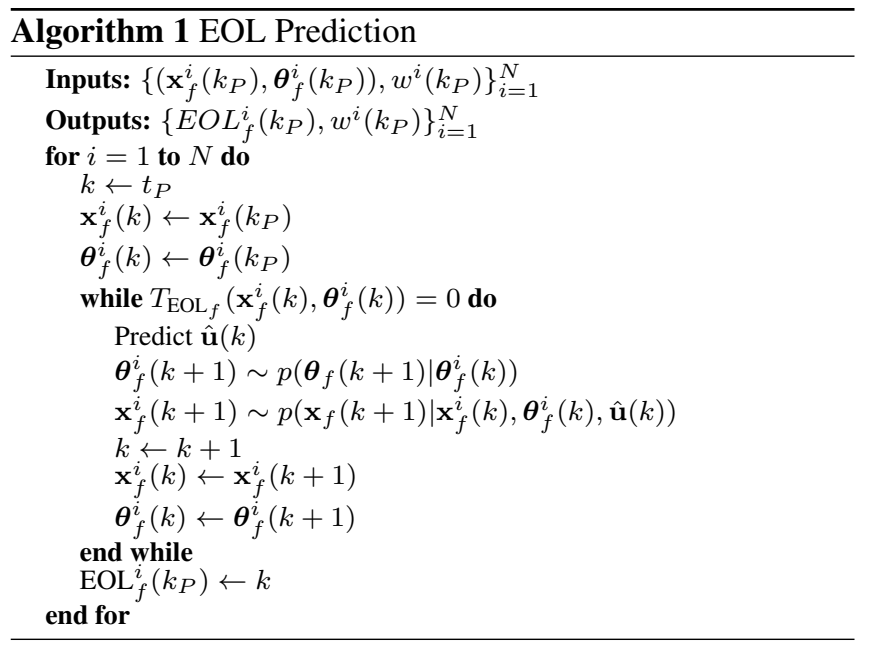

\section{Prediction}

The prediction module is invoked at time $k_{P}$ to predict the EOL and/or RUL of the component for each hypothesized fault, $f$. Specifically, using the current joint stateparameter estimate, $p\left(\mathbf{x}_{f}\left(k_{P}\right), \boldsymbol{\theta}_{f}\left(k_{P}\right) \mid \mathbf{y}\left(0: k_{P}\right)\right)$, which represents the most up-to-date knowledge of the system at time $k_{P}$, the goal is to compute $p\left(\operatorname{EOL}_{f}\left(k_{P}\right) \mid \mathbf{y}\left(0: k_{P}\right)\right)$ and $p\left(\operatorname{RUL}_{f}\left(k_{P}\right) \mid \mathbf{y}\left(0: k_{P}\right)\right.$. As described in detail in [12], we assume the state-parameter distribution is represented as a discrete set of weighted samples, i.e.,

$$
\begin{aligned}
p\left(\mathbf{x}_{f}\left(k_{P}\right), \boldsymbol{\theta}_{f}\left(k_{P}\right) \mid \mathbf{y}\left(0: k_{P}\right)\right) & \approx \\
& \sum_{i=1}^{N} w^{i}\left(k_{P}\right) \delta_{\left(\mathbf{x}_{f}^{i}\left(k_{P}\right), \boldsymbol{\theta}_{f}^{i}\left(k_{P}\right)\right)}\left(d \mathbf{x}_{\mathbf{f}}\left(k_{P}\right) d \boldsymbol{\theta}_{f}\left(k_{P}\right)\right),
\end{aligned}
$$

where $i$ denotes the index of a single sample, $w^{i}$ is the weight of this sample, and $\delta$ represents the Dirac delta function located at $\left(\mathbf{x}_{f}^{i}\left(k_{P}\right), \boldsymbol{\theta}_{f}^{i}\left(k_{P}\right)\right)$.

Similarly, we can approximate the EOL as

$$
\begin{aligned}
& p\left(\mathrm{EOL}_{f}\left(k_{P}\right) \mid \mathbf{y}\left(0: k_{P}\right)\right. \approx \\
& \sum_{i=1}^{N} w^{i}\left(k_{P}\right) \delta_{\mathrm{EOL}_{f}^{i}\left(k_{P}\right)}\left(d \mathrm{EOL}_{f}\left(k_{P}\right)\right) .
\end{aligned}
$$

The general approach to solving the prediction problem is through simulation. Each sample is simulated forward to EOL to obtain the complete EOL distribution. The pseudocode for the prediction procedure is given as Algorithm 1 [7]. Each sample $i$ in the state-parameter distribution is propagated forward until $T_{\mathrm{EOL}_{f}}\left(\mathbf{x}_{f}^{i}(k), \boldsymbol{\theta}_{f}^{i}(k)\right)$ evaluates to 1 , at which point EOL has been reached for this particle, and the EOL prediction is weighted by the weight of the sample at $k_{P}$.

Note that we need to hypothesize future inputs of the system, $\hat{\mathbf{u}}(k)$, for prediction, since fault progression is dependent on the operational conditions of the system. The choice of expected future inputs depends on the knowledge of expected operational settings. 
Table 1. Fault signatures for selected faults and measurements.

\begin{tabular}{ccccccccccccc}
\hline Faults & $q_{\text {Pump2 }}$ & $q_{\text {Filt1 }}$ & $q_{\text {Filt2 }}$ & $q_{\text {Pump4 }}$ & $q_{\text {ROPump }}$ & $q_{\text {Prod }}$ & $p_{\text {WT }}$ & $p_{\text {FT1 }}$ & $p_{\text {FT2 }}$ & $p_{\text {Prod }}$ & $p_{\text {Filt1 }}$ & $p_{\text {Filt2 }}$ \\
\hline$R_{\text {Filt1 }}^{-}$ & $0-$ & $0-$ & $0-$ & $0-$ & 00 & 00 & 00 & $0+$ & $0-$ & 00 & $0+$ & $0-$ \\
$R_{\text {Filt2 }}^{-}$ & 00 & 00 & $0-$ & $0-$ & 00 & 00 & 00 & 00 & $0-$ & 00 & 00 & $0+$ \\
$A_{\text {FO }}^{-}$ & 00 & $0-$ & $0-$ & $0-$ & $0-$ & 00 & 00 & 00 & $0+$ & 00 & 00 & $0-$ \\
$A_{\mathrm{RO}}^{-}$ & 00 & 00 & 00 & 00 & $0-$ & $0-$ & 00 & 00 & 00 & $0-$ & 00 & 00 \\
$q_{\text {Filt1 }}^{(b, 20)}$ & 00 & +0 & 00 & 00 & 00 & 00 & 00 & 00 & 00 & 00 & 00 & 00 \\
$q_{\text {Filt2 }}^{(d, 0.1)}$ & 00 & 00 & $0+$ & 00 & 00 & 00 & 00 & 00 & 00 & 00 & 00 & 00 \\
$p_{\text {Prod }}^{(b, 2)}$ & 00 & 00 & 00 & 00 & 00 & 00 & 00 & 00 & 00 & +0 & 00 & 00 \\
$q_{\text {ROPump }}^{(d, 0.01)}$ & 00 & 00 & 00 & 00 & $0+$ & 00 & 00 & 00 & 00 & 00 & 00 & 00 \\
\hline
\end{tabular}

\section{EXPERIMENTAL RESULTS}

This section presents the results of our diagnosis and prognosis experiments on the simulation model of the WRS shown in Fig. 2. For these experiments, as mentioned in Section 2, we selected eight different faults, namely $R_{\mathrm{Filt} 1}^{-}, R_{\mathrm{Filt} 2}^{-}, A_{\mathrm{FO}}^{-}, A_{\mathrm{RO}}^{-}$, $q_{\text {Filt1 }}^{(b, 20)}, q_{\text {Filt1 }}^{(d, 0.1)}, p_{\text {Prod }}^{(b, 2)}$, and $q_{\text {ROPump }}^{(d, 0.01)}$. Table 1 provides the fault signature table for the selected faults and measurements of the WRS. Note that sensor faults affect only the signature for the faulty sensor. Parametric faults such as the clogging of filters and membranes cause more than one sensor to deviate from nominal.

For the purposes of prognosis, the EOL of the WRS is defined by when the filters need to be replaced. This is indicated by when the differential pressures across the individual filters, $p_{\text {Filt1 }}$ or $p_{\text {Filt2 }}$ cross a certain pressure threshold, $p_{\text {Filt } 1} \uparrow$ or $p_{\text {Filt2 }} \uparrow$. Hence, $T_{\mathrm{EOL}_{f}}=1$ if $p_{\text {Filt1 }} \geq p_{\text {Filt1 }} \uparrow$ or $p_{\text {Filt2 }} \geq p_{\text {Filt } 2} \uparrow$.

In our experiments, for fault detection, we use the simulation model of the nominal system to generate nominal system behavior. The fault signatures for faults considered in our experiments and the WRS measurements are given in Table 1, and used for fault isolation. For fault identification, we adopt particle filtering [9] as our observer. Particle filtering is the most general estimation scheme as it can be applied to nonlinear systems with arbitrary probability distributions for process and measurement noise that can be nonlinearly coupled with the states. Particle filtering is a sequential Monte Carlo sampling method for Bayesian filtering and approximates the belief state of a system using a weighted set of samples, or particles. Each particle consists of an instantiation of values of the state vector, and describes a possible system state. As observations are obtained, each particle is moved stochastically to a new state using the nominal state transition function, and the weight of each particle is readjusted to reflect the likelihood of that observation given the particle's new state. We assume all random variables to be Gaussian.

We now present a detailed integrated diagnosis and prognosis scenario to illustrate our approach. In this scenario, Filter 2 clogging begins at $t=0$ min according to Eqn. 31 with wear rate $\Delta R_{\text {Filt2 }}=-5 \times 10^{-12}$. A fault is detected at $309 \mathrm{~min}$, via an increase in the differential Filter 2 pressure, $p_{\text {Filt2 }}$ (see Fig. 5). As shown in Table 1, only fault $R_{\text {Filt2 }}^{-}$has a $0+$ signature for $p_{\text {Filt2 }}$, indicating that the fault $R_{\text {Filt2 }}^{-}$would cause the pressure $p_{\text {Filt2 }}$ to increase. Since this is the only fault consistent with the observed deviation, a singleton fault

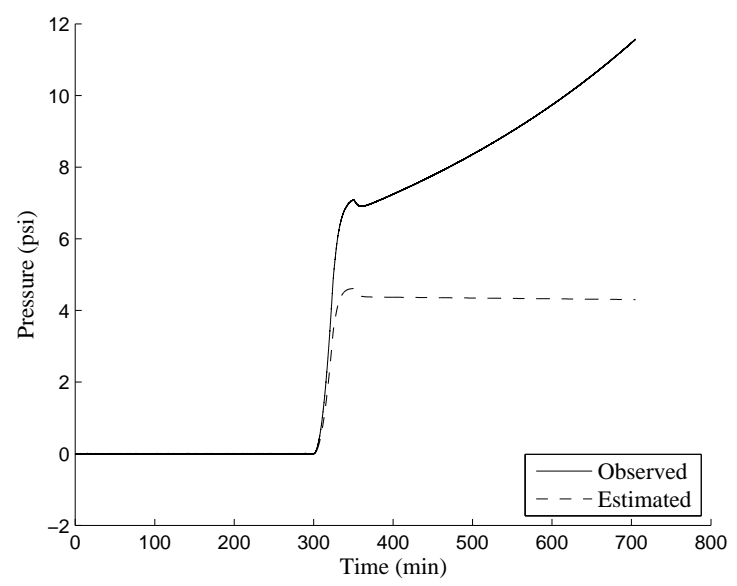

Figure 5. Estimated and observed values of sensor $p_{\text {Filt2. }}$.

candidate set, $\left\{R_{\text {Filt } 2}^{-}\right\}$, is generated, and the fault is detected and isolated at the same time.

Fault identification is initiated once the number of fault candidates was reduced to three or less (i.e., $\sigma=3$ ) by the qualitative isolator, or if the qualitative isolator has executed for $p=400 \mathrm{~min}$. For our particular problem, we found $N=50$ particles sufficient for accurate tracking, and used $\Delta k^{\max }=0$ for each observer used for fault identification. For the Filter 2 Clogging fault, the wear rate $\Delta R_{\text {Filt2 }}$ estimate averages to $\Delta R_{\text {Filt } 2}=-5.11759 \times 10^{-12}$ with small output error (see Fig. 6). The corresponding RUL predictions, made at an interval of $10 \mathrm{~min}$ from the time the fault identifier converges to a solution are shown in Fig. 7 which plots the predicted RUL [13] of the WRS under $R_{\mathrm{Filt} 2}^{-}$from $t=$ $540 \mathrm{~min}$ at $10 \mathrm{~min}$ intervals. As mentioned in Section 3, at each prediction point, Fig. 7 shows true RUL, RUL*, and a probability density function of the predicted RUL represented using its median value and the $5-25 \%$ and $75-95 \%$ ranges. The plot also shows a cone of $\alpha=10 \%$ accuracy around RUL predictions. From the first prediction point, at $t=540 \mathrm{~min}$, the algorithm has converged and the median RUL predictions remain within the accuracy window of $10 \%$ except at $t=$ $610 \mathrm{~min}, t=620 \mathrm{~min}$, and $t=640 \mathrm{~min}$. In order to make predictions, we assume that the future inputs are known. Hence, the uncertainty in the predictions is due solely to that resulting from the identification stage, and explains why all RUL predictions did not fall within the accuracy cone. In our simulation experiments, for illustrative purposes, we chose 


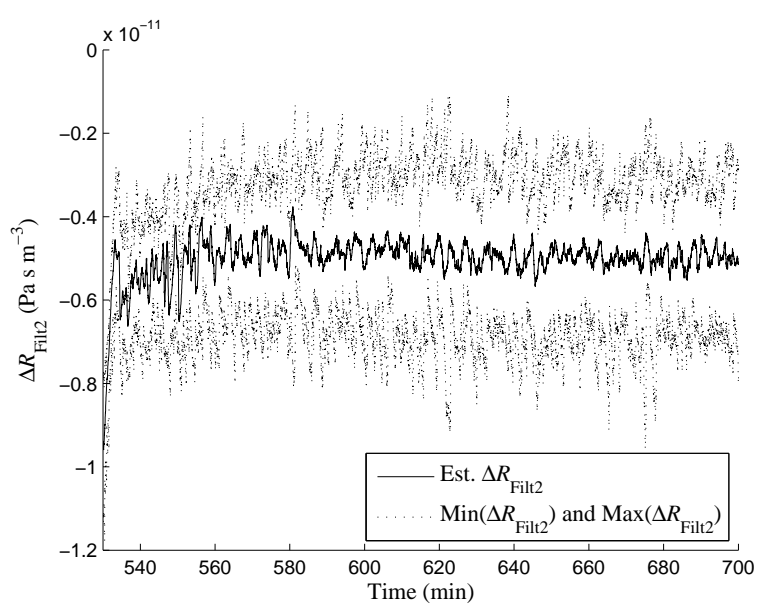

Figure 6. Estimated $\Delta R_{\text {Filt2 }}$ values.

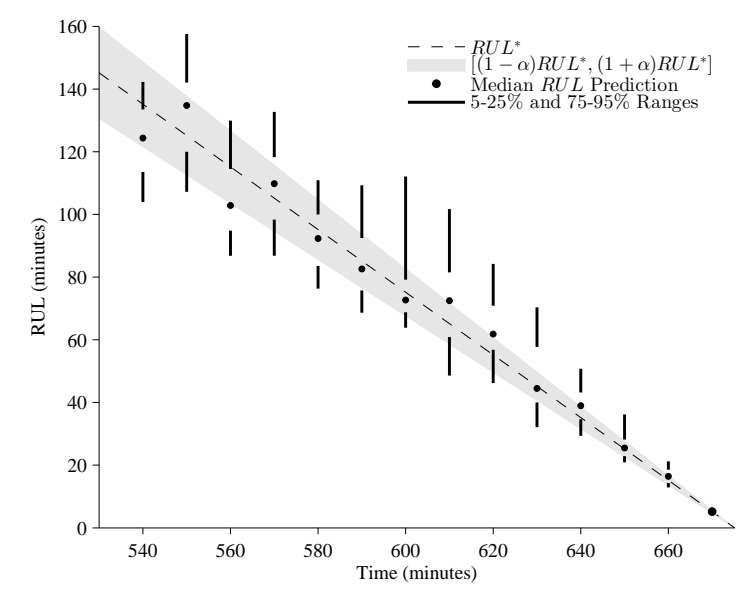

Figure 7. Predicted RUL of the WRS under $R_{\mathrm{Filt} 2}^{-}$fault. The median is indicated with a dot and confidence intervals of $5 \%$ and $95 \%$ by lines. The gray cone depicts an accuracy requirement of $\alpha=10 \%$.

$\alpha=10 \%$. In real-world scenarios, however, the value of $\alpha$ flows down from the top-level requirements [14].

\section{Simulation Results}

Table 2 summarizes the detection and isolation results of several simulation experiments. The columns of the table represent the true fault; true injected value of the fault parameter; $t_{f}$, the time of fault occurrence in minutes from the start of experiment; $\Delta t_{d}$, the time in minutes to detect the fault; $\Delta t_{i}$, the time in minutes for qualitative isolation to reduce the candidate set as much as possible; and the set of fault candidates after qualitative fault isolation. Given the small number of faults, in each of the experiments, the observed measurement deviation resulted in a singleton fault candidate set to be generated (with the true fault being the only fault candidate). As a result the fault was detected and isolated at the same time, and hence, $\Delta t_{i}=\Delta t_{d}$ for these experiments. Note that this is typically not the case in large systems with many possible faults, where more than one measurement deviation is needed to isolate the true singlefault candidate. Once the sensor faults are correctly isolated and identified, the sensor readings can be "corrected", and hence, the presence of this type of sensor faults do not
Table 2. Diagnosis Results

\begin{tabular}{llllll}
\hline True Fault & $\begin{array}{l}\text { True Fault } \\
\text { Magnitude }\end{array}$ & $\begin{array}{l}t_{f} \\
(\mathrm{~min})\end{array}$ & $\begin{array}{l}\Delta t_{d} \\
(\mathrm{~min})\end{array}$ & $\begin{array}{l}\Delta t_{i} \\
(\mathrm{~min})\end{array}$ & $\begin{array}{l}\text { Fault } \\
\text { Candidates }\end{array}$ \\
\hline Nominal & N/A & N/A & $\infty$ & $\infty$ & $\emptyset$ \\
$R_{\text {Filt1 }}^{-}$ & $-1.00 \times 10^{-12}$ & 1.00 & 60.67 & 60.67 & $R_{\text {Filt1 }}^{-}$ \\
$R_{\text {Filt2 }}^{-}$ & $-5.00 \times 10^{-12}$ & 1.00 & 309.37 & 309.37 & $R_{\text {Filt2 }}^{-}$ \\
$A_{\text {FO }}^{-}$ & $-1.60 \times 10^{-11}$ & 326.00 & 232.70 & 232.70 & $A_{\text {FO }}^{-}$ \\
$A_{\text {RO }}^{-}$ & $-2.00 \times 10^{-10}$ & 326.00 & 37.87 & 37.87 & $A_{\text {RO }}^{-}$ \\
$q_{\text {Filt1 }}^{(b, 20)}$ & 20.00 & 175.00 & 0.03 & 0.03 & $q_{\text {Filt1 }}^{(b, 20)}$ \\
$q_{\text {Filt2 }}^{(d, 0.1)}$ & 0.10 & 400.00 & 0.90 & 0.90 & $q_{\text {Filt2 }}^{(d, 0.1)}$ \\
$p_{\text {Prod }}^{(b, 2)}$ & 2.00 & 410.00 & 0.02 & 0.02 & $p_{\text {Prod }}^{(b, 2)}$ \\
$q_{\text {ROPump }}^{(d, 0.01)}$ & 0.01 & 404.00 & 8.67 & 8.67 & $q_{\text {ROPump }}^{(d, 0.01)}$ \\
\hline
\end{tabular}

cause the system to violate the constraints of acceptable behavior. Hence, for sensor faults, prognosis is not applicable since we assumed a fault mode that manifests itself without measurable precursors. The prognosis results for the $R_{\text {Filt2 }}^{-}$ have already been presented above. In our experimental runs, the slowly progressing filter and membrane blockage faults take between $37.87 \mathrm{~min}$ and $309.37 \mathrm{~min}$ to be detected. The sensor faults however are detected and isolated more quickly, between $0.02 \mathrm{~min}$ and $8.67 \mathrm{~min}$.

\section{Conclusions}

This paper applied an integrated model-based diagnostic and prognostic framework to a WRS designed to serve as a testbed for long duration testing of next generation spacecraft WRS for human spaceflight missions. Our approach made use of a common modeling paradigm to model both the nominal and faulty system behavior, and we successfully demonstrated diagnosis and prognosis results on the WRS.

As part of future work, we are planning to analyze the realworld experimental data from the WRS at the Sustainability Base to refine our WRS simulation model. We also plan to extend the model by including the modeling of the mass flow conservation of solute and solvent molecules. Since the WRS qualifies as a complex system, improvements in efficiency and scalability can be achieved by running distributed diagnosis and prognosis algorithms on this system [15]. Finally, we will investigate the effect of relaxing the single fault assumption and extend our approach to diagnosis and prognosis of multiple faults in the WRS.

\section{ACKNOWLEDGMents}

The funding for this work was provided by the Habitat Systems component of the NASA Autonomous Systems (AS) Project. The authors would also like to thank Dr. Mark Schwabacher for his support and thank Dr. Michael Flynn and Dr. Matthew Daigle for the insightful discussions with regards to this work.

\section{REFERENCES}

[1] K. D. Pickering, K. Wines, G. M. Pariani, L. A. Franks, J. Yeh, B. W. Finger, M. L. Campbell, C. E. Verostko, C. Carrier, J. C. Gandhi, and L. M. Vega, "Early results 
of an integrated water recovery system test," in Proc. of the 29th International Conference on Environmental Systems, 2001.

[2] S. Gormly, V. D. Adams, T. Cath, A. Childress, M. Flynn, and E. Beaudry, "Direct osmotic concentration: A primary water treatment process of space life support applications," in Proc. of the 33rd International Conference on Environmental Systems, 2003.

[3] M. Flynn, M. Hightower, L. Delzeit, S. Gormly, M. Hammoudeh, H. Shaw, T. Richardson, and K. Howard, "Sustainability base graywater recycling system." AIAA-2011-5145, 41st International Conference on Environmental Systems, Portland, Oregon, 2011.

[4] NASA Ames Research Center Sustainability Base, http://www.nasa.gov/externalflash/sustainability-base/.

[5] I. Roychoudhury and M. Daigle, "An integrated modelbased diagnostic and prognostic framework," in Proc. of the 22nd International Workshop on Principles of Diagnosis, 2011.

[6] I. Roychoudhury, "Distributed diagnosis of continuous systems: Global diagnosis through local analysis," Ph.D. dissertation, Vanderbilt University, 2009.

[7] M. Daigle and K. Goebel, "Multiple damage progression paths in model-based prognostics," in Proceedings of the 2011 IEEE Aerospace Conference, Mar. 2011.

[8] T. Cath, A. Childress, and M. Elimelech, "Forward osmosis: Principles, applications, and recent developments," Journal of Membrane Science, vol. 281, no. 1, pp. 70-87, 2006.

[9] M. S. Arulampalam, S. Maskell, N. Gordon, and T. Clapp, "A tutorial on particle filters for online nonlinear/non-Gaussian Bayesian tracking," IEEE Transactions on Signal Processing, vol. 50, no. 2, pp. 174-188, 2002.

[10] M. J. Daigle, I. Roychoudhury, G. Biswas, and X. Koutsoukos, "A comprehensive diagnosis methodology for complex hybrid systems: A case study on spacecraft power distribution systems," IEEE Trans. on System, Man, and Cybernetics, Part A, vol. 4, no. 5, pp. 917 - 931, September 2010.

[11] P. J. Mosterman and G. Biswas, "Diagnosis of continuous valued systems in transient operating regions," IEEE Transactions on Systems, Man, and Cybernetics, Part A: Systems and Humans, vol. 29, no. 6, pp. 554$565,1999$.

[12] M. Daigle and K. Goebel, "A model-based prognostics approach applied to pneumatic valves," International Journal of Prognostics and Health Management, vol. 2, no. 2, Aug. 2011

[13] A. Saxena, J. Celaya, B. Saha, S. Saha, and K. Goebel, "Metrics for offline evaluation of prognostic performance," International Journal of Prognostics and Health Management, 2010.

[14] A. Saxena, I. Roychoudhury, J. Celaya, B. Saha, S. Saha, and K. Goebel, "Requirement flowdown for prognostics health management," in Proceedings of the AIAA InfotechAerospace, 2012.

[15] M. Daigle, A. Bregon, and I. Roychoudhury, "A distributed approach to system-level prognostics," in Proceedings of the Annual Conference of the Prognostics and Health Management Society 2012, 2012, pp. 7182 .

\section{BIOGRAPHY}

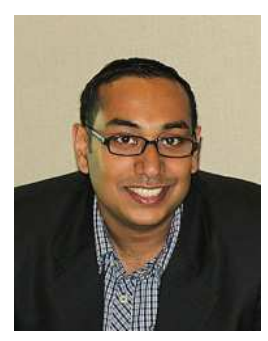

Indranil Roychoudhury received the B.E. (Hons.) degree in Electrical and Electronics Engineering from Birla Institute of Technology and Science, Pilani, Rajasthan, India in 2004, and the M.S. and Ph.D. degrees in Computer Science from Vanderbilt University, Nashville, Tennessee, USA, in 2006 and 2009, respectively. Since August 2009, he has been with SGT, Inc., at NASA Ames Research Center as a Computer Scientist. Dr. Roychoudhury is a member of the Prognostics and Health Management Society and the IEEE. His research interests include hybrid systems modeling, model-based diagnostics and prognostics, distributed diagnostics and prognostics, and Bayesian diagnostics of complex physical systems.

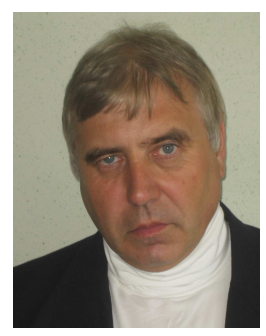

Vasyl Hafiychuk is a senior research scientist with SGT, Inc. He obtained his BS, MS and PhD in physics at Moscow Institute of Physics and Technology (PhysTech) working on nonlinear physics problems. He is an author of more than 100 publications. He worked as a senior scientific researcher at the Academy of Science in Ukraine; professor at Krakow University of Technology, Poland; CityTech CUNY NYC; and Middlesex County College, NJ. His research interests include applied mathematics, nonlinear dynamics and self-organization, structural mechanics, and acoustics.

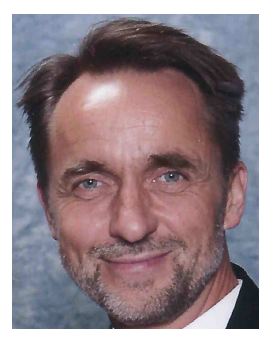

Kai Goebel is Deputy Area Lead of the Discovery and Systems Health Technology Area at NASA Ames Research Center. He also coordinates the Prognostics Center of Excellence. Prior to joining NASA in 2006, he was a senior research scientist at General Electric Corporate Research and Development center since 1997. Dr. Goebel received his Ph.D at the University of California at Berkeley in 1996. He has carried out applied research in the areas of real time monitoring, diagnostics, and prognostics and he has fielded numerous applications for aircraft engines, transportation systems, medical systems, and manufacturing systems. He holds 17 patents and has co-authored more than 250 technical papers in the field of IVHM. Dr. Goebel was an adjunct professor of the CS Department at Rensselaer Polytechnic Institute (RPI), Troy, NY, between 1998 and 2005 where he taught classes in Soft Computing and Applied Intelligent Reasoning Systems. He has been the co-advisor of 6 Ph.D. students. Dr. Goebel is a member of several professional societies, including ASME, AAAI, AIAA, IEEE, VDI, SAE, and ISO. He was the General Chair of the Annual Conference of the PHM Society, 2009, has given numerous invited and keynote talks and held many chair positions at the PHM conference and the AAAI Annual meetings series. He is currently member of the board of directors of the PHM Society and associate editor of the International Journal of PHM. 